\title{
La política criminal del nudge. Algunos desafíos del Análisis Conductista a las teorías del Derecho Penal tradicionales
}

\author{
Criminal-nudge-policy. Behavioral Analysis Challenges to \\ Traditional Criminal Law Theory ${ }^{*}$
}

Diego Moreno Cruz ${ }^{* *}$

"Nello scendere le scale
Ci metto più attenzione
Sarebbe imperdonabile
Giustiziarmi sul portone
Proprio nel giorno in cui
La decisione è mia
Sulla condanna a morte
O l'amnistia"

Il bombarolo; F. De Andrè

Recepción: 06/01/2019

Evaluación: 13/02/2019

Aceptación final: 31/07/2019

Resumen: La objeción de Harel y Teichman en contra de que los gobiernos sigan las "recomendaciones de política pública provenientes de los teóricos conductistas", para llevar a la gente a obedecer las reglas penales y, de este modo, para prevenir la ocurrencia de crímenes, puede ser entendida como cuestiones acerca de la permisibilidad ética y la utilidad práctica de aplicar

* Traducción del inglés de Ricardo Arenas Ávila.

** Doctor en filosofía del derecho, profesor en la misma disciplina y docente-investigador del Centro de Estudios en Filosofía y Derecho de la Universidad Externado de Colombia, Bogotá, Colombia. Correo electrónico: diego.moreno@uexternado.edu.co. Agradezco a Ricardo Arenas Ávila por la traducción del inglés al español de este escrito. 
lo que llamaré "política criminal del nudge". Mi propósito en este escrito no es ofrecer una definición exhaustiva de este tipo de política pública ni tampoco justificar su uso desde una perspectiva ética, sino tan solo enunciar algunos retos a las teorías tradicionales del derecho penal en las que Harel y Teichman asientan su objeción.

Palabras clave: análisis económico-conductista, políticas públicas criminales, nudge, prevención.

\begin{abstract}
The objection raised by Harel and Teichman about following "policy recommendations prescribed by behavioral theorists" by governments, that are intended to lead people to obey penal rules and, hence, to prevent crimes from occurring, may perfectly well be understood as both ethical permissibility and practical usefulness concerns about applying what I will call "criminal-nudging-policies". My purpose in this paper it is not to offer an exhaustive definition of this type of public policy neither to justify their use from an ethical point of view, but to point out some challenges to traditional theories of criminal law from which Harel and Teichman raised such theoretical issues.
\end{abstract}

Keywords: behavioral economics analysis, criminal public policies, nudge, deterrence.

\title{
1. Introducción
}

En su ensayo, Harel y Teichmann (HT) intentan contener el entusiasmo propagado de los gobiernos por seguir las "recomendaciones de política pública" formuladas desde la perspectiva del "Análisis económico-conductista del Derecho" (AECD), en asuntos de derecho penal y de política criminal: es decir, un tipo de acción de política pública que consiste en aplicar herramientas-tácticas-técnicas, que están diseñadas sobre la base de los hallazgos de experimentos psicológicos acerca de cómo funciona el aparato cognitivo humano, con la intención de dirigir a la gente a obedecer las normas penales $y$, de este modo, a prevenir la ocurrencia de delitos.

Ellos afirman que el éxito futuro del AECD en asuntos de derecho penal y política criminal depende del grado en el que los partidarios de esta perspectiva logren lidiar con la carga de justificar la aplicación de las herramientas conductistas, en relación con cada uno de los tres problemas 
siguientes: (1) las tesis asumidas por el AECD sobre la racionalidad de las personas y la justificación del derecho penal y de la sanción penal son incongruentes con las tesis que al respecto son asumidas por las teorías jurídicas penales tradicionales: específicamente el AECD es incongruente con la teoría comunicativa de Duff; (2) la fuerza predictiva del AECD se ve afectada por el hecho de que, con base en una hipótesis explicativa de la ciencia psicológica y cognitiva del comportamiento humano, los partidarios del AECD ofrecen hipótesis diferentes, $\mathrm{y}$ a veces contradictorias, sobre los resultados (probables) de la aplicación de tales herramientas en asuntos de control criminal: en otras palabras, hay una indeterminación sobre los efectos probables de aplicar este tipo de instrumentos de política pública en contextos jurídico-penales; y (3) estas herramientas son inútiles si no se alinean con los juicios intuitivos y no-consecuencialistas, las razones no económicas y la perspectiva de la gente sobre la aplicación de penas y de la política criminal por parte del Estado (por ejemplo, juicios acerca de la rectitud del sistema de justicia penal).

En este artículo, ofreceré una serie de razones teóricas para justificar el uso de los instrumentos de control del AECD, a saber, intentaré superar los problemas planteados por HT para contener el entusiasmo que los gobiernos han manifestado por este tipo de herramientas. En primer lugar, identificaré algunas afirmaciones imprecisas en la reconstrucción que ellos hacen de la metodología del AECD, por cuanto está basada en una serie de ambigüedades. Procederé a sugerir algunas aclaraciones y, de este modo, especificaré cuál es el objeto de su objeción: i.e., la "política criminal del nudge"1: una clase de acción de los gobiernos que está informada comportamentalmente y que es realizada con la intención de controlar la acción criminal de las personas por medio de una elección consciente del regulador (architect) sobre la "arquitectura" de los problemas de decisión de la gente -“choice architecture"-, es decir, la enmarcación del problema de decisión o la "puesta en escena" del contexto en el que las personas eligen obedecer o desobedecer normas penales (Sección 2). En segundo

1 A pesar de que el término "nudging" no aparece en su ensayo, los problemas teóricos planteados por ellos pueden ser entendidos perfectamente como preocupaciones de permisibilidad ética y utilidad práctica sobre la aplicación de los nudges en asuntos de política criminal. 
lugar, mostraré que la segunda cuestión planteada por ellos, que llamaré el "problema metodológico", no es, de hecho, un problema para el AECD, sino un problema general para el control social a través del derecho. Y, en una última parte, ofreceré razones para superar las cuestiones primera y tercera planteadas por HT, que llamaré el "problema normativo" (Sección 3). Para concluir, desde la perspectiva de las teorías cognitivas de la psicología humana y del comportamiento humano, señalaré algunos desafíos a las tradicionales teorías del derecho penal.

\section{AECD y la elección de la arquitectura}

HT reconstruyen la aproximación del AECD confrontándolo con el Análisis Económico del Derecho (AED). Ambos enfoques ofrecen prescripciones o recomendaciones a los gobiernos sobre cómo controlar el comportamiento humano. Sin embargo, la reconstrucción que HT hacen del AECD es imprecisa en dos puntos que requieren algunas aclaraciones para evitar malentendidos sobre cuál es el objeto de los problemas planteados por ellos.

(1) La primera afirmación imprecisa de su reconstrucción es que el AED asume que la gente decide qué acción realizar basada en el costo y los beneficios de los cursos de acción a su disposición, y que el AECD, en contraste, toma en cuenta que las decisiones y acciones de las personas son influenciadas también por sus creencias y preferencias. En otras palabras, HT afirman que el AED no toma en cuenta las creencias y las preferencias de la gente al hacer sus recomendaciones de política pública.

Sin embargo, de hecho, tanto el AED como el AECD asumen que las personas están provistas de una racionalidad instrumental -o de medio a fin- para resolver sus problemas de decisión; ambas perspectivas también asumen que las personas deciden basadas en un análisis de costo-beneficio que sería imposible de advertir sin asumir que las personas forman en su mente creencias y preferencias; $y$, por último, ambos enfoques analíticos se basan, cada uno de distintas maneras, en el modelo de razonamiento instrumental de creencias-preferencias que subyace a la "teoría de la decisión racional".

Según la teoría de la decisión racional, un problema de decisión está conformado por una serie de acciones alternativas, resultados y circuns- 
tancias. La evaluación de probabilidades (grados de creencia) de las circunstancias relevantes del mundo -que el agente considera podría afectar los resultados atados a cada uno de los actos opcionales- y de los valores (o preferencias) de cada uno de los resultados riesgosos son representados por números que determinan conjuntamente un estimado de utilidad para cada uno de los cursos de acción alternativos bajo consideración ${ }^{2}$. La utilidad de cada acción es equivalente al valor asignado a los resultados alternativos atados a la acción opcional, sopesados por su probabilidad ${ }^{3}$. El valor del resultado y de las probabilidades asignadas a las circunstancias son subjetivas en el sentido en que reflejan respectivamente las preferencias y las creencias de la persona, sin consideración de las razones externas (morales o fácticas) para creerlas y preferirlas ${ }^{4}$.

El AED se basa en la teoría de la utilidad esperada como una teoría lógica "that prescribes how decisions should be made and as description of how Econs make choices"5: escoger el acto con la utilidad máxima estimada, es decir, la acción que produce un resultado que es al menos tan bueno al de cualquier otro de los resultados posibles. Por su parte, el AECD se fundamenta en una teoría cognitiva de la toma de decisiones humanas que intenta describir "how Humans actually make risky choices, without assuming anything about their rationality"6: una teoría psicológica cognitiva sobre cómo la racionalidad instrumental de la gente funciona en realidad. Esta teoría muestra que los Humans se separan de manera sistemática de la teoría (económica) tradicional de la decisión racional en los dos sentidos o usos que dan los economistas a esta teoría: como una serie de proposiciones axiomáticas y lógicas para asignar correctamente valores a resultados y probabilidades a las circunstancias del mundo, y, por lo tanto, como una serie de proposiciones descriptivas del proceso de toma de decisión de los Econs ${ }^{7}$.

Al tratarse de una descripción del proceso de toma de decisión de los Humans, la teoría cognitiva ofrece una serie de proposiciones psicológicas

2 Jeffrey, R., The Logic of Decision, Chicago, University of Chicago Press, 1985, p. 1.

3 Kahneman, D., Thinking Fast and Slow, London, Penguin Books, 2011, p. 274.

4 Jeffrey, R., Op. cit., p. 1.

5 Kahnemann, D., Op. cit., p. 270.

6 Ibid, p. 271.

7 Ibid, p. 270. 
que explican por qué la gente se comporta sistemáticamente y, por ende, de manera predecible, de una forma tal que es, a los ojos de un Econ, instrumentalmente incorrecta para maximizar su propia utilidad. La identificiación de acciones de las personas que son desviaciones sistemáticas de la teoría de la decisión racional - de los axiomas y proposiciones acerca de cómo toman decisiones los Econs - se llaman "sesgos" (biases). Se trata de efectos que son explicados y predichos sobre la base de la identificación de la aplicación sistemática de "heurísticas" en el razonamiento práctico de toma de decisión de las personas: es decir, la aplicación de atajos mentales que reducen la complejidad y los costos relacionados con la concentración y el tiempo requerido para recolectar y evaluar información, a saber, para fijar la serie de acciones alternativas y asignar tanto valores a los resultados como probabilidades a las circunstancias del mundo; o como simples reacciones afectivas (e incoscientes) a la enmarcación del problema de elección, o al diseño del contexto en el cual ellas toman decisiones, sin importar cualquier propósito consciente de las personas de ahorrar costos cognitivos y tiempo ${ }^{8}$. La mayoría de las veces, esos atajos mentales producen un resultado correcto, es decir, funcionan bien. Sin embargo, también es cierto que estas heurísticas otras veces tienen por efecto que las personas cometan sistemática y, en consecuencia, predeciblemente errores: la tendencia de formar preferencias y creencias que los llevan a elegir una acción que es inadecuada para maximizar su propia utilidad; es decir, una acción que sería descartada por la evaluación correcta de valores y probabilidades hechas por un Econ.

De modo que, la identificación de heurísticas y de los sesgos cognitivos es útil para explicar y predecir el comportamiento de las personas.

La teoría cognitiva es buena describiendo, explicando y prediciendo el comportamiento humano, pero no es una teoría normativa para indicar cómo evaluar de manera correcta la información disponible ni para guiar el comportamiento de las personas. Y también es cierto que la teoría de la decisión racional tradicional es buena indicando cómo evaluar correc-

8 Tversky, A. y Kahneman, D., "The framing of decisions and the psychology of choice", Science, 211, 4481, 1981, p. 458. 
tamente valores y probabilidades, pero no es buena ni describiendo ni guiando el comportamiento de las personas ${ }^{9}$. Basado en las explicaciones y predicciones de la teoría psicológica cognitiva, el AECD ofrece una serie de prescripciones encaminadas a corregir o utilizar los sesgos cognitivos de los seres humanos, para lograr los objetivos normativos del sistema jurídico: a saber, las razones que justifican por qué un tipo de acto es modulado deónticamente por prohibiciones (por ejemplo, un tipo de acto criminal), obligaciones y permisiones jurídicas. De modo que, el AECD no puede ser considerado como una teoría sino como una serie de prescripciones políticas encaminadas a hacer efectiva la obediencia de la ley y, en consecuencia, a alcanzar, con el grado más alto de efectividad, una o más de las distintas metas normativas del sistema jurídico.

En este sentido, una de las preguntas principales que el AECD ha tratado de responder es cómo lograr que los objetivos normativos del sistema jurídico puedan compaginar con lo que es, o podría ser, conocido sobre la psicología humana. Con esta cuestión en mente, el AECD prescribe políticas que son formuladas sobre la base de los descubrimientos de las ciencias conductistas, es decir, una serie de explicaciones/predicciones con base en las cuales el AECD complementa o socava las respuestas que el AED ha dado a esta pregunta teórica ${ }^{10}$. Los partidarios del AECD afirman que las recomendaciones de política pública que se sirven de los hallazgos de los experimentos de psicología cognitiva son más precisas y confiables que las prescripciones políticas del AED, que no están comportamentalmente informadas, es decir, políticas que no consideran el trasfondo de la mente de las personas -el Background: elementos mentales involuntarios, a saber, reacciones afectivas, capacidades, habilidades, hábitos ${ }^{11}$-, que condiciona e informa tanto la intención formada deliberada o conscientemente, como aquella formada inconscientemente: las acciones de política pública de los gobiernos prescritas por el AED no toman en cuenta el trasfondo mental de las personas, que es un presupuesto necesario de la actividad mental humana

9 Ídem.

${ }^{10}$ Sunstein, C., Kahneman, D. y Schkade, D., "Assessing Punitive Damages (with Notes on Cognition and Valuation in Law)", The Yale Journal, 107, 1998, 2071-2153, pp. 2080-2081.

${ }^{11}$ Searle, J., Making the Social World. The Structure of Human Civilization, Oxford, OUP, 2010, pp. 31-32. 
para actuar intencionalmente de una y no de otra forma posible. Una mayor precisión descriptiva de la toma de decisiones de seres humanos lleva a una mayor confiabilidad predictiva del comportamiento humano y, en consecuencia, a una mayor efectividad de las intervenciones del gobierno en el cumplimiento de las metas normativas del sistema normativo ${ }^{12}$.

Para resumir, el AED y el AECD están basados en la "teoría de la decisión racional”, pero cada una de estas perspectivas de análisis lo hace de distintas maneras. Para formular sus recomendaciones de política pública, el AED usa esta teoría como una teoría (normativa) sobre cómo decide comportarse un Econ y, de este modo, sobre cómo debería comportarse un Human para tomar decisiones "racionalmente" correctas. Por su parte, el AECD se basa en la teoría de la decisión racional con objetivos descriptivos y explicativos: es decir, la usa con el objetivo de mostrar y explicar desviaciones sistemáticas de esta teoría por parte de los Humans en la toma de decisiones. Por lo tanto, el AECD basa sus recomendaciones de política pública en una "teoría cognitiva de la toma de decisión" que utiliza la teoría de la decisión racional de Econs como un punto de referencia de corrección, para identificar las desviaciones sistemáticas de las elecciones y acciones realizadas por los Humans. De este modo, una teoría que identifica sesgos cognitivos, para explicar y predecir el comportamiento de los seres humanos. En consecuencia, tanto el AED como el AECD toman en cuenta las creencias-preferencias de los decisores para prescribir acciones de política pública a los gobiernos.

(2) La segunda afirmación imprecisa de la reconstrucción de HT consiste en que, según ellos, los partidarios del AECD consideran que el regulador influencia las decisiones de las personas al cambiar "los modos en que el mundo aparenta ser sin cambiar el modo en el que el mundo es". Sin embargo, un argumento importante de los partidarios del AECD es, precisamente, que "cómo creemos que el mundo es" es un resultado de una "choice architecture"13. Veámos.

12 Jolls, C., Sunstein, C.R. \& Thaler, R., "A Behavioral Approach to Law and Economics", Stanford Law Review, 50, 1998, pp. 1471-1550.

13 Thaler, R. \& Sunstein, C., Nudge: Improving Decisions about Health, Wealth and Happiness, London, Penguin Books, 2009, 6, 11, pp. 20-24, 56-57, 83-102; Sunstein, C., The Ethics of 
La política criminal del nudge.

Cada decisión presupone una "choice arquitecture", es decir, "the background conditions for people's choices" 14 : el modo en que las opciones, circunstancias y resultados de los problemas de decisión son presentados, enmarcados o estructurados; la forma, o el diseño, del contexto en el cual las personas escogen uno y no otro de los cursos de acción opcionales. Así pues, "a choice architecture will nudge" el comportamiento de las personas en el sentido en que las creencias y las preferencias de la gente sobre cuál acción realizar dependen de la elección de la arquitectura del problema de decisión. En consecuencia, el nudge es generalizado y ubicuo, ya sea porque la elección de la arquitectura es tomada consciententemente, o bien porque ella es un resultado de un proceso psicológico inconsciente o, simplemente, del azar" ${ }^{15}$ : "There is no getting away from this"16.

De acuerdo con esto, es razonable considerar que el derecho " $[. .$.$] crea-$ tes some form of choice architecture"17, una elección que está dirigida a dar forma a "[...] individuals' lives and the common culture" por medio de sanciones e incentivos (positivos) ${ }^{18}$.

Sin embargo, el nudge es entendido como una intervención consciente de los reguladores en la actividad mental de las personas, que consiste en cambiar levemente la arquitectura del problema de decisión, y que apunta a hacerlas reaccionar de un modo que sea replicable y predecible en aras de obtener efectivamente resultados sociales que sean beneficiosos. Un grado mínimo de interferencia que no prohíbe ninguna opción - es decir, que no reduce la serie de opciones disponibles - o impone a la gente costos económicos significativos, o esfuerzos cognitivos irrazonables, para elegir "salirse" "cheaply and easy" del nudge $e^{19}$. En consonancia, bien se puede afirmar que el nudge es un típico ejercicio intencional del poder por parte

Influence. Government in the Age of Behavioral Science, Cambridge, New York, Cambridge University Press, 2016, cap. 2.

14 Sunstein, C., The Ethics of Influence, op. cit., p. 22.

15 Waldron, J., "It's All for Your Own Good", The New York Review of Books, oct. 9, 2014.

${ }^{16}$ Waldron, J., Op. cit.; Sunstein, C., The Ethics of Influence, Op. cit., pp. 36-37, 76.

${ }^{17}$ Sunstein, C., The Ethics of Influence, Op. cit., p. 20; Sunstein, C., "Misconceptions about Nudges", Journal of Behavioral Economics for Policy, 2, 1, 2018, p. 62.

18 Green, L., "Introduction", en Hart, H., The Concept of Law, Oxford, OUP, 2012, p. xxxii.

19 Yeung, K., "Nudge as Fudge", The Modern Law Review, 75, 1, 2012, 122-148, 129; Thaler, R. y Sunstein, C., Op. cit., p. 9. 
de los gobiernos ${ }^{20}$, que está encaminado “[...] to steer people in particular directions but also allow them to go their own way"21. Por lo tanto, los nudges son diferentes al tipo de acción intencional de los gobiernos, que si bien está comportamentalmente informada, y es realizada conscientemente, cambia la elección de la arquitectura de la gente prohibiéndoles alguna opción e imponiéndoles incentivos significativos: por ejemplo, "mandates, ban and incentives" 22 . Por el contrario, el nudge "is a policy intervention for redirecting an agent's choices by very slightly altering their choice conditions so that the interference is kept to a minimum"23.

Los gobiernos pueden utilizar intencionalmente su poder de control social para alterar el comportamiento de la gente en contextos de decisión particulares y, de este modo, lograr diferentes objetivos sociales de los sistemas jurídicos a través de una variedad de nudges.

Algunos nudges conducen a la gente en la dirección en la que ellos quieran ir, otros nudges dirigen a las personas en la dirección en la que los arquitectos quieren que la gente vaya. Algunos nudges se utilizan para prevenir que la gente se lastime con sus propias acciones. Otros se utilizan para prevenir que la gente lastime a otras personas ${ }^{24}$. Algunos nudges están hechos para mejorar tanto el bienestar social como los intereses individuales de los decisores, otros nudges están hechos para obtener tan solo el primer resultado y no el segundo ${ }^{25}$. Algunos nudges (por ejemplo, etiquetas, advertencias y recordatorios) contrarrestan sesgos comportamentales (por ejemplo, el sesgo del presente y el sesgo optimista ${ }^{26}$ ), otros nudges (por

${ }^{20}$ Searle, J., Op. cit., p. 155.

${ }^{21}$ Sunstein, C., The Ethics of Influence, Op. cit., p. 21.

${ }^{22}$ Sunstein, C., "Misconceptions about Nudges", Op. cit., p. 65.

${ }^{23}$ Sin embargo, el nudge es un concepto equívoco que está lejos de tener un significado claro: se utiliza en sentidos distintos, e incluso contradictorios, al respecto véase Mongin, P., Cozic, M., "Rethinking Nudge: not One but Three Concepts", Behavioural Public Policy, 2, 1, 2018, p. 107-124.

${ }^{24}$ Sunstein, C., The Ethics of Influence, Op. cit., p. 32.

25 Yeung, K., Op. cit., pp. 123-124.

${ }^{26}$ Sunstein, C., "Misconceptions about Nudges", Op. cit., p. 64: el sesgo del presente se refiere a la tendencia de la gente de darle más peso a sus deseos presentes que a los resultados beneficiosos a largo plazo. El sesgo optimista se refiere a la tendencia de que nuestras acciones llevan a resultados que terminan siendo mejores para nosotros en comparación a los resultados malos obtenidos por dicha acción, mostrados por la información estadística. Si 
La política criminal del nudge.

ejemplo, las "default rules") funcionan gracias a la presencia de sesgos (por ejemplo, la inercia y la procrastinación), y otros se encaminan a facilitarle a la gente llegar a dónde quieran ir, sin considerar sesgo comportamental alguno, por ejemplo, la revelación de información relevante al tomador de decisiones ${ }^{27}$.

Algunos de estos nudges apuntan a las capacidades deliberativas de la gente para hacerlos decidir activamente, o conscientemente, un curso de acción particular, basados en el contenido de significado de la información del problema de decisión, a saber, los “Sistem 2 nudges". Estos son los nudges encaminados a informar o educar a la gente para que aprendan a decidir mejor: por ejemplo, las etiquetas, advertencias, recordatorios, etc. Otros nudges se enfocan en la actividad mental cognitiva automática, irreflexiva e intuitiva de la gente para llevarlos a decidir un curso de acción particular, como una reacción inconsciente a los estímulos producidos en su mente por la forma en que se enmarca el problema de decisión, y sin considerar el contenido de significado de la información: nudges que no están encaminados a informar o a educar, sino a contrarrestar sesgos o a utilizar una heurística (inalterable) para producir un resultado social deseable. En estos casos, la gente se comporta de cierta manera sin saber, o sin ser consciente de que lo que han elegido hacer, o no hacer, es un resultado de la influencia que el nudge ejerce en sus elecciones. Las "default rules" son un ejemplo de este tipo de nudges. Son nudges que no aumentan la agencia humana ni la elección activa de la gente, es decir, "Sistem 1 nudges"28.

Así que, si es cierto que las creencias y las preferencias que las personas forman en su mente son un resultado de la "eleccion de la arquitectura", la afirmación de HT de que los partidarios del AECD abogan por prescripciones que manipulan las elecciones de la gente podría ser entendida como la aserción de que ellas consisten en recomendaciones a los gobiernos de mentir a la gente sobre la información de los problemas de decisión o sobre cuál es el contexto de su elección. Si este es el sentido de su afirmación,

fumo es porque creo que no me pasará nada malo. Los nudges contrarrestan estos sesgos al enfatizar los riesgos a largo plazo de fumar.

27 Ídem.

28 Sunstein, C., The Ethics of Influence, Op. cit., p. 122, 149-153. 
es algo que, en caso de ser cierto, sería una actitud mala y reprochable por parte de los reguladores, como también lo sería todas las acciones de política pública de los gobiernos que sean o estén basadas en una mentira, para hacer que la gente obedezca el derecho. En este sentido, esta es una objeción ética incuestionable ${ }^{29}$.

Sin embargo, utilizar la elección de la arquitectura de la gente no significa necesariamente que la acción de política pública consista en una mentira: ni cuando los nudges son físicos -nudges que funcionan gracias al sesgo de la ilusión en la percepción de la gente: por ejemplo, pintar líneas en las carreteras para crear la ilusión (creencia falsa) de que el vehículo está aumentando su velocidad, con el propósito de que los conductores reduzcan la velocidad en zonas (por ejemplo, zonas escolares) donde existe el riesgo de causar algún daño a los otros usuarios de la vía- ni tampoco cuando los nudges son psico-físicos - nudges que funcionan según la forma en que se enmarque el problema de decisión- ${ }^{30}$.

\subsection{Política criminal del Nudge}

El derecho penal es un sistema coercitivo que moldea el contexto de elección de la gente, al prohibirles escoger ciertos cursos de acción disponibles. Las reglas penales son "órdenes respaldadas por amenzaas" que son "la expresión de un deseo de que otros hagan o se abstengan de hacer ciertas cosas". A las personas que tienen razones de mayor peso para desobedecer las normas "la sanción adosada a la regla les proporciona un motivo para dicha adecuación" ${ }^{31}$. De modo que, las reglas penales son resultado de la elección de la arquitectura del problema de decisión de la gente: es decir, una acción que es realizada por los gobiernos con la intención de que las reglas penales sean obedecidas, apelando a la capacidad-de-elección-activa de las personas para que actúen del modo deseado por el arquitecto, es

\footnotetext{
${ }^{29}$ Ibíd, pp. 3, 60.

30 Yeung, K., Op. cit., pp. 132-133.

31 Hart, H., El concepto de derecho, Buenos Aires, Abeledo Perrot, 1963, pp. 53 y 49.
} 
La política criminal del nudge.

decir, a la capacidad de las personas de considerar las reglas como razones (morales o prudenciales) para actuar.

Las reglas penales son instrumentos coercitivos de control social que "[...] express and channel social power"32 a través de su contenido de significado, esto es, como razones para actuar, y también a través de otras formas que son independientes del contenido de significado de las reglas, pero que de igual manera son utilizadas para conducir a la gente a obedecerlas.

Para obtener un resultado social beneficioso, el legislador puede expedir una norma penal basándose en las ciencias conductistas: es decir, utilizar la forma en que se formulan las reglas como un tipo de herramienta informada comportamentalmente para lograr objetivos jurídicos. Sin embargo, las reglas penales son instrumentos que alteran el comportamiento de las personas al prohibir un tipo de acto y al imponer (des)incentivos significativos para (des)obedecer lo que el derecho requiere que ellas hagan, o se abstengan de hacer, a saber, cometer una acción típica (token-act) de un tipo de acción objeto de criminalización (type-act). Por lo tanto, una regla penal no es un nudge en un sentido estricto; controlar el comportamiento de las personas por medio de mandatos soportados en amenazas no es una instanciación del tipo de control "nudging".

A pesar de esto, es posible aplicar un nudge a la gente para llevarlos a obedecer las normas penales, por medio del nudging penal, esto es, un tipo de acción informada comportamentalmente que está encaminada a alterar el comportamiento de las personas al apelar a los estímulos producidos en su mente, y que mantiene la libertad de la gente de obedecer o de encarar, con cierto grado de probabilidad, una pena que la norma misma conecta a un suceso de desobediencia. Estas intervenciones en la mente de las personas son elecciones conscientes hechas, ya sea por los legisladores a través de la enmarcación de la norma penal de cierta manera particular, o por los creadores de políticas a través de la configuración del contexto en el cual las personas eligen obedecer o desobedecer la norma penal. Ambos tipos de intervención son nudges penales utilizados para evitar la ocurrencia de delitos, a saber, intervenciones que utilizan la elección de la arquitectura de la elección de la gente para prevenir que lastimen a otros, y no el tipo de

${ }^{32}$ Green, L., Op. cit., xxxii. 
nudges encaminados a prevenir que las personas se lastimen a sí mismas con sus propias acciones.

Por una parte, se puede aplicar un tipo de nudge para que la gente obedezca el derecho al apelar a su actividad cognitiva intuitiva, esto es, a las reacciones inconscientes de la gente a los estímulos producidos por el diseño de la norma jurídica misma, sin considerar su contenido de significado como una razón para actuar. Por ejemplo, se ha demostrado que la gente reacciona diferente a enunciados normativos que expresan la misma norma jurídica, es decir, que tienen el mismo contenido de significado, pero que son formuladas o enmarcadas en términos modales deónticos diferentes: "You are prohibited from carrying out more than one of the following actions"; "You are permitted to carry out only one of the following actions". El primer enunciado "You are prohibited" le facilita más a la gente saber qué acción es impermisible que el segundo enunciado "You are permitted"33.

Por otra parte, existen nudges penales que ejercen su influencia por medio de técnicas de control social que no consisten en la forma en que se enmarcan o formulan las reglas: pequeños cambios en algún aspecto de la elección de la arquitectura del contexto de decisión de la gente (de obedecer

33 "You are permitted to carry out only one of the following actions:

Take the apple or the orange, or both.

Take the pear or the orange, or both.

Are you permitted to take the orange?"

"You are prohibited from carrying out more than one of the following actions:

Take the apple or the orange, or both.

Take the pear or the orange, or both.

Are you permitted to take the orange?"

La respuesta lógica a ambos problemas es "no". Si usted toma la naranja, está llevando a cabo dos acciones. La gente reacciona diferente si el problema se enmarca en términos de permisión o prohibición, y las personas reaccionan correctamente más fácilmente a la segunda enmarcación que a la primera, basada en modelos mentales que la gente construye como representaciones mentales del contenido de significado de los enunciados normativos. Estas reacciones distintas muestran que las personas cometen errores de inferencia en el razonamiento deóntico, es decir, "ilusiones deónticas”, Bucciarelli, M. \& Johnson-Laird, P.N. "Naïve Deontics: A Theory of Meaning, Representation, and Reasoning”, Cognitive Psychology, 50, 2, 2005, pp. 159-193, 183-188. 
o desobedecer una regla penal) para llevarlos a actuar de conformidad con lo que el derecho requiere que hagan o se abstengan de hacer.

Hay nudges que apuntan a la capacidad deliberativa o activa de elección de la gente. Por ejemplo, las campañas de educación pública, la propaganda, que son utilizadas para transmitir un mensaje que hace explícitas las razones que subyacen a la criminalización de una acción-tipo, es decir, las razones de por qué desobedecer una norma penal es considerado como moralmente incorrecto por la sociedad; para informar sobre datos estadísticos cuidadosamente recolectados sobre las detecciones y condenas de cierta clase de acciones delictivas, para contrarrestar los sesgos optimistas de la gente o para protegerlos del engaño o la manipulación producidos por la elección consciente de la arquitectura por parte de arquitectos privados; es decir, nudges que son usados para contrarrestar los nudges realizados por personas (no reguladores) para conducir a la gente a cometer delitos.

Existen nudges que funcionan al apelar a la actividad cognitiva automática de la gente, es decir, hacen que la gente se comporte de cierta manera como una reacción a los estímulos producidos en su mente por el modo en que el problema de decisión es presentado a las personas e independientemente de cualquier razón para actuar. Algunos de estos nudges utilizan la heurística que la gente ya sigue para guiarlos hacia la obediencia del derecho, por ejemplo, al reportar algún evento de detección y/o condena de un crimen con la intención de desencadenar la "availability heuristic" que lleva a la gente a sesgos predecibles en sus juicios, a saber, un nudge que funciona porque la gente sobreestima la frecuencia y la relevancia de eventos basándose en la disponibilidad de la memoria de recuperar fácilmente instanciaciones de una clase de sucesos. Una cobertura extensa de los medios de estos sucesos provoca en la mente de las personas la creencia de que casos de ese tipo han ocurrido más veces que las indicadas por datos, o consideraciones, de estadística relevantes. No es casualidad que los regímenes autoritarios se preocupen tanto por cuáles sucesos deben ser cubiertos por los medios y cuáles eventos no deben ser reportados ${ }^{34}$.

Dicho lo anterior, los nudges prescritos por los partidarios del AECD pueden ser entendidos como un conjunto de enunciados deónticos uti-

34 Kahnemann, D., Op. cit., pp. 59-70. 
lizados como directivas o reglas técnicas a ser seguidas por parte de los gobiernos en el proceso de creación de políticas públicas, esto es, reglas que indican "the means to be used for the sake of attaining a certain end" - por ejemplo, cómo utilizar la elección de la arquitectura para prevenir la ocurrencia de un delito-, y que presuponen una "proposición anankástica"35, es decir, la verificación de una explicación y predicción del comportamiento de las personas en contextos de elección particulares, que están basadas en hallazgos conductistas de experimentos de laboratorio de la psicología cognitiva. La confiabilidad y precisión de la regla técnica, por ejemplo, "si usted, gobierno, quiere incrementar el porcentaje de pago de impuestos, esto es, evitar que la gente evada sus obligaciones tributarias, entonces seleccione la lista de contribuyentes al azar al principio del año", lo que presupone la proposición anankástica "si (no) selecciona la lista de los contribuyentes al azar al principio del año, en efecto, el porcentaje de evasión de impuestos (no) disminuirá".

\section{Problemas teóricos de los nudges penales}

HT argumentan que el éxito futuro del AECD en asuntos de derecho penal y de política criminal depende del grado en el que sus partidarios lidien con la carga de justificación creada por tres problemas que pueden ser reformulados como cuestiones normativas y metodológicas concernientes a la aplicación de la "política criminal del nudge" para prevenir la ocurrencia de delitos: 1) la incongruencia entre el AECD y las teorías penales tradicionales sobre la racionalidad de la gente y las justificaciones de la pena; 2) la indeterminación de los efectos probables de aplicar un " $n u d g e$ " en contextos de elección jurídico-penales; 3) utilizar nudges penales para prevenir delitos que no esten en consonancia con los juicios intuitivos no-consecuencialistas, las razones no económicas y la perspectiva de la gente sobre la pena y la política criminal relacionadas con ciertos delitos.

35 Von Wright, G., Norm and Action. A logical Enquiry, London, Routledge and Kegan Paul, 1963, pp. 9-11, 101. 
Primero, analizaré la cuestión (2), es decir, el problema metodológico. Segundo, analizaré los problemas (1) y (3), es decir, el problema normativo: (1) tiene que ver con la presunción sobre la racionalidad de la gente al (des) obedecer las normas penales, y (3) con las razones que la gente tiene, en general, para creer que las acciones de política pública de los gobiernos para controlar el comportamiento de los criminales son (im)permisibles

\subsection{El problema metodológico}

No es posible estar en desacuerdo con la afirmación de HT de que "es necesario a este punto mayor investigación empírica del comportamiento criminal real que corrobore, refute o refine las hipótesis expuestas en la literatura conductista" ${ }^{36}$. Pero lo cuestionable de su argumento es la sugerencia de que esto es una necesidad práctica exclusiva de las recomendaciones de política criminal al ser el derecho penal un área peculiar. El problema de indeterminación metodológica que TH señalan está relacionado en realidad con toda clase de políticas públicas. Por tanto, este problema no puede ser un desafío a la aplicación del AECD para analizar asuntos de política criminal. Más bien, el problema de la indeterminación que TH señala implica el deber técnico de prestar atención a las predicciones/explicaciones empíricas en la toma de decisiones y en la realización de acciones de política pública, así como en analizar la acción de las personas como si ellas fueran irracionales.

Por otra parte, si la afirmación de HT de que la gente tiene razones no-económicas para justificar el control criminal -a saber, la tercera cuestión planteada por ellos- está basada en hipótesis empíricas de experimentos de psicología cognitiva, entonces tal afirmación adolece del mismo problema metodológico de indeterminación planteado por ellos sobre la aplicación de nudges penales.

Por lo tanto, el problema metodológico no es desafío a la justificación de las prescripiciones del AECD, sino un desafío de la justificación del control social y de la administración gubernamental del derecho en general.

\footnotetext{
${ }^{36}$ Harel, A. y Teichman, D., Op. cit., sección 4.3.
} 


\subsection{El problema normativo}

Los nudges son herramientas de política pública encaminadas a (re)dirigir a la gente a seguir el derecho, ya sea apelando a sus capacidades deliberativas para escoger activamente, o bien, apelando a su proceso de elección psicológico irreflexivo e inconsciente. En el último caso, la gente no es consciente de que su elección es el resultado de dicha intervención: una reacción a los estímulos que la intervención produce en su mente. El nudge es un ejemplo del tipo de prescripción política del AECD.

Así, el entusiasmo del AECD por el nudging penal asume una racionalidad de medio-a-fin de las personas y presupone una justificación instrumental del derecho penal y de la imposición de penas.

Por una parte, cuando la gente enfrenta el problema de elegir entre obedecer o desobedecer lo que el derecho requiere que se haga, o se abstenga de hacer, las personas escogen la opción que a su juicio sea, en comparación con las opciones alternativas, un medio adecuado para lograr lo que ellos ya quieren, prefieren o valoran. Escoger desobedecer el derecho expresa la disposición de una persona de asumir el riesgo de sufrir un castigo, previa y cuidadosamente advertido a ella por la norma penal, basándose en su evaluación subjetiva de los valores de los resultados alternativos y de las probabilidades asignadas a las circunstancias que condicionan la ocurrencia de cada uno de los resultados riesgosos.

Por otra parte, el nudging penal se justifica para promover la obediencia del derecho. Un creador de políticas públicas informado por las ciencias conductistas sabe que la gente utiliza heurísticas que producen sesgos predecibles en sus juicios, a saber, errores sistemáticos al evaluar los valores de los resultados y las probabilidades de las circunstancias del mundo; también sabe que la elección de la arquitectura del problema de decisión de las personas es útil para contrarrestar, o utilizar, sesgos cognitivos para dirigir a la gente a obedecer el derecho; también sabe que puede aplicar nudges que persuaden a las personas -por ejemplo, campañas de educación pública- al hacer explícita la justificación moral de por qué un tipo o clase de acción es objeto de criminalización por parte del legislador y por qué se justifica castigar a aquellos que eligen cometer típicos actos de ese tipo de acción, es decir, nudges que apelan al sistema 2, esto es, a razones para actuar y a 
la capacidad deliberativa de las personas de escoger activamente; también sabe que puede aplicar nudges que conduzcan a las personas a actuar de conformidad con lo que exigen las normas penales apelando al sistema 1, es decir, a las reacciones cognitivas inconscientes a los estímulos producidos por la forma en que es presentado el problema de decisión, y sin ofrecer a las personas razones para actuar conforme al derecho: de modo que, las personas son inconscientes de que ellas eligen obedecer el derecho por la influencia que el nudge ejerce en sus elecciones.

Ambos son nudges encaminados a prevenir la ocurrencia de delitos. A pesar de lo anterior, para obtener este propósito el nudging criminal tiene limitaciones de carácter ético-normativo. Estas intervenciones en la mente de las personas deben ser ejercidas sin limitar o cambiar las opciones de elección disponibles (es decir, obedecer o desobedecer), y sin imponer costos significativos (económicos o cognitivos) en los decisores, es decir, que ellos puedan fácilmente, $y$ con costos insignificantes, optar por escoger un curso de acción diferente (desobedecer) a aquella acción preferida por el arquitecto (obedecer).

TH argumentan que la política criminal del nudging, que es defendida por los partidarios del AECD, está en conflicto tanto con la presunción de la racionalidad de las personas como con la justificación de la pena de las “teorías de derecho penal tradicionales", más precisamente, con las teorías deontológicas tradicionales retribucionistas, y entre estas últimas, en especial, la teoría de la comunicación racional del proceso penal de Duff (1), y también que las presunciones instrumentales del AECD son incongruentes con los juicios intuitivos no-consecuencialistas, las razones no económicas y la perspectiva de la gente sobre la la pena y la política criminal (2).

(1) La finalidad del derecho penal no es controlar el comportamiento humano e inducir a la gente a actuar de conformidad con las normas penales, con el propósito de prevenir (o evitar) la ocurrencia de delitos, sino, más bien, persuadir a la gente de actuar racionalmente de conformidad con tales normas (HT). Es decir, las personas merecen ser tratadas como seres humanos racionales.

El AECD percibe a la gente como seres humanos malos: las personas (des)obedecen el derecho basados en la probabilidad de ser castigados, es decir, motivados por las reglas que están soportadas por amenazas, y no 
por la razón de que cometer un típico acto del tipo de acción criminalizado por una norma penal es moralmente incorrecto. Las normas penales son razones morales para actuar, y el derecho penal se justifica para hacer que las personas las sigan como guía de su conducta, a saber, normas que no son proferidas para ser meramente observadas por razones prudenciales. Por lo tanto, desde esta aproximación, la racionalidad de la gente no es instrumental -"no es acerca de cuál es la mejor manera de promover sus intereses"-, sino una racionalidad informada moralmente: la gente es percibida como seres humanos con la capacidad de hacer elecciones autónomas basadas en lo que es "lo correcto y lo incorrecto, lo moral y lo inmoral"37.

Este problema de incongruencia normativa es ilustrado por HT confrontando el AECD con la "Teoría de la comunicación de Duff” (la “curial perspective" 38 ).

El propósito central del derecho penal es llamar al sospechoso de la comisión de un delito a rendir cuentas ante los jueces penales. Los juicios son una oportunidad para entablar "discurso racional entre quien ha cometido un delito y el Estado"39. El proceso ocupa el lugar central no solo del derecho procesal penal, sino también del derecho penal sustancial ${ }^{40}$. El proceso da la oportunidad a los defendidos de ofrecer razones tanto para negar la acusación en contra de ellos como para argumentar una defensa ${ }^{41}$. Los procedimientos por los cuales esta comunicación racional es posible tienen un valor intrínseco: pasar de la atribución de responsabilidad a la imposición merecida y justa de una pena como un resultado de una discusión racional moral entre el sospechoso y el estado ${ }^{42}$. El propósito principal del derecho penal no es imponer una pena justificada, sino determinar si el presunto delincuente es responsable por su comportamiento y castigarlo

${ }^{37}$ Harel, A. \& Teichman, D., Op. cit., sección 3.

${ }^{38}$ Edwards, J., "Theories of Criminal Law", en Edward N. Zalta (ed.), The Stanford Encyclopedia of Philosophy, Stanford, Stanford University, 2018, disponible en: https://plato.stanford. edu/archives /fall2018/entries/criminal-law/

${ }^{39}$ Harel, A. \& Teichman, D., Op. cit., sección 3.

${ }^{40}$ Edwards, J., Op. cit.

41 Ídem.

42 Duff, R., "Responsabilidad y punibilidad en el derecho penal" en Kramer M., Grant C., Colburn, B. \& Hatzistavrou, A. (eds.), El legado de H. L. A. Hart. Filosofía jurídica, política y moral, Madrid, Marcial Pons, 2012, pp. 145-168. 
si no ofrece razones exculpantes "adecuadas" por lo que hizo. Y, lo más importante, el juicio tiene la función de provocar en el delincuente el reconocimiento de que ha realizado una acción reprochable y que merece sufrir un castigo por ello. Asimismo, si es el caso de que se prueben condiciones exculpantes, el proceso tiene la función de desencadenar el reconocimiento del resto de la sociedad de que el defendido no merece ser castigado por su comportamiento ${ }^{43}$. En consecuencia, el propósito principal del derecho penal se cumple incluso si la pena no se impone en el defendido como resultado del juicio ${ }^{44}$.

La principal razón de HT para afirmar este problema teórico de incongruencia normativa es que desde la perspectiva de las teorías tradicionales del derecho penal, "El derecho penal busca esclarecer de manera racional si un ilícito ha tenido lugar y, en su caso, importa que el criminal reconozca que ha cometido un ilícito". El criminal es percibido "como un ser racional que necesita ser educado y transformado", y no como una máquina de estímulos que es susceptible de ser engañada, y que es fácilmente embaucable y manipulable. Por lo tanto, "explotar los errores cognitivos y el comportamiento irracional" para prevenir el crimen es una acción de política pública fraudulenta y manipuladora, que trata a la gente como "autómatas", a saber, "como bestias potencialmente peligrosas que deben ser controladas explotando sus debilidades"45.

(2) Basados en hipótesis empíricas, HT afirman que lo que es asumido acerca de la naturaleza de la racionalidad de las personas y las justificaciones instrumentales acerca del derecho penal presupuestas por AECD son incongruentes con los juicios intuitivos no-consecuencialistas, las razones no-económicas y las opiniones de la gente sobre las penas y las políticas criminales; hipótesis que funcionan para reforzar de contera las creencias teóricas de las teorías retribucionistas de la pena.

Si se comete un acto típico del tipo de acción que está prohibida por una regla general, entonces el delincuente merece sufrir la consecuencia jurídica que tal regla conecta, imputa, a la comisión de actos de dicho tipo;

${ }^{43}$ Ibíd., pp. 155 y ss.

${ }^{44}$ Ibid., p. 148.

${ }^{45}$ Las citas en este párrafo son de Harel, A. \& Teichman, D., Op. cit., sección 3. 
en otras palabras, si sucesos de violación de la norma han ocurrido, se justifica castigar al culpable del injusto. La pena se justifica porque el delincuente merece sufrir por el injusto-culpable que cometió $-\mathrm{y}$ “the suffering must be proportionate to the wrongdoer's guilt" 46 -, pero no se justifica hacer sufrir al que cometió el injusto ni para disuadir a la gente de cometer actos típicos ni para inocuizar al delincuente para que no pueda cometer delitos futuros por el tiempo en el que estará privado de su libertad, por ejemplo, en prisión. La única razón para imponer el sufrimiento punitivo en un criminal es que ella lo merece. En otras palabras, el castigo justificado debe ser impuesto por razones de merecimiento (la "punitive view" ${ }^{37}$ ).

Asimismo, para otras teorías de la retribución, la amenaza de una pena advertida por las normas penales expresa un significado moral — por ejemplo, la desaprobación de las acciones criminales por parte de la comunidad; aquellos que cometen un crimen merecen ser castigados-y, por tal razón, las normas penales no son reducibles a meros incentivos negativos, o motivaciones, para obedecer el derecho.

Este enfoque teórico es consistente con los hallazgos empíricos sobre las opiniones no-económicas, las intuiciones no-consecuencialistas de la gente acerca de la justificación de la pena. El quantum de las sanciones no depende de la probabilidad de detección. La aplicación de la pena no es para prevenir la ocurrencia del delito, sino para infligir un sufrimiento justamente merecido en quien ha cometido un injusto, si y solo si, esa pena es acorde con las intuiciones morales de la gente sobre la rectitud del sistema de justicia penal. Si este se desvía de lo que son las intuiciones morales (no-consecuencialistas) de las personas sobre qué es lo justo, entonces la gente tiene razones para desobedecer lo que el derecho penal requiere de ellos, y, por lo tanto, la eficacia de la obediencia del derecho disminuye.

De este modo, es posible concluir que si las normas penales y las políticas de control criminal no están en consonancia con las intuiciones no-consecuencialistas y las opiniones de la gente sobre la pena y la política criminal (al menos, en relación con algunos delitos), aplicar un nudge penal no haría ninguna diferencia práctica en la actividad mental de las personas.

\footnotetext{
${ }^{46}$ Gardner, J., Op. cit., p. xvi, nota 7.

${ }^{47}$ Edwards J., Op. cit.
} 
Por estas razones, desde las teorías de derecho penal deontológicas, entre estas, la teoría de la comunicación racional en el proceso de Duff, el nudging penal podría ser considerado un tipo de acción política reprochable e impermisible: es impermisible utilizar la imposición de sufrimiento a un delincuente como una elección de la arquitectura consciente del juez, o de cualquier otra autoridad decisora, con la intención de contener a otros, y a quien está siendo castigado, de cometer delitos futuros.

\subsubsection{Un falso problema}

El problema de la incongruencia normativa planteado por HT se basa en una ambigüedad: ellos no diferencian la influencia del derecho ejercida a través de las normas penales como razones para actuar y las herramientas, tácticas, utilizadas por el gobierno para hacer que la gente obedezca las normas penales (1). Además, su argumento falla en dos sentidos: el AECD y las teorías del derecho penal tradicionales de la retribución (en especial, la teoría de la comunicación racional en el proceso) son incomparables porque cada una de estas aproximaciones tienen una naturaleza distinta, y se refieren a distintos problemas y funciones del derecho penal (2), y, además, estas funciones no son inconsistentes la una con la otra; a saber, la justificación instrumental de la aplicación de una pena es consistente con las justificaciones no-instrumentales de la imposición al delincuente de una pena justamente merecida como resultado del proceso, como lo aceptaría una teoría mixta del derecho penal, por ejemplo, bajo cierta interpretación, la teoría deontológica de la prevención de Hart (3).

(1) El problema normativo planteado por HT es ambiguo sobre el control de la acción humana a través de reglas y de la aplicación de políticas públicas: esto es, este desafío a las recomendaciones del AECD se refiere a distintas formas en el que el derecho influencia en la mente humana. Dirigir las personas a que obedezcan el derecho a través de normas penales es diferente a conducirlas a obedecer estas normas por medio de estrategias o tácticas de administración gubernamental, es decir, instrumentos de política pública que ejercen influencia en las personas por medios distintos a las normas penales en cuanto razones para actuar. 
Para obtener resultados sociales "beneficiosos" a la luz de uno, o más, objetivos normativos del sistema jurídico, el derecho se encamina a alterar el comportamiento de las personas de forma predecible: un "medium" para influenciar con las normas jurídicas sus mentes ${ }^{48}$. Esta afirmación presupone aceptar que para que el derecho sea una herramienta efectiva, su obediencia debería obtenerse a través de normas penales que puedan ser seguidas por personas como razones morales o prudenciales para actuar. Sin embargo, las normas jurídicas no tienen la capacidad de hacer que sean obedecidas por medio de ellas mismas. Entonces, en contextos de elección particulares de personas particulares (por ejemplo, il bombarolo), el derecho es muchas veces objeto de la administración gubernamental. En otras palabras, a veces son utilizadas tácticas-técnicas-instrumentos de política pública diferentes al contenido de significado de las normas jurídicas -i.e. como razones prudenciales o morales para actuar- para llevar a la gente a hacer o abstenerse de hacer lo que el derecho requiere de ellas.

El nudging penal consiste precisamente en aplicar herramientas de administración del derecho, es decir, una clase de acción de política pública que hace referencia al ejercicio intencional de los gobiernos de su poder de control social sobre la actividad psicológica de las personas, para obtener una obediencia efectiva del derecho, a saber, para prevenir que la gente desobedezca normas penales por medio de instrumentos diferentes a las normas jurídicas como razones para actuar.

Por lo tanto, el problema normativo planteado por HT concierne a la obedeciencia de las normas penales como razones para actuar -en el derecho penal se trata de hacer que la gente actúe racionalmente de conformidad con lo que las normas penales requieren que ellos hagan, o se abstengan de hacer; la gente obedece el derecho si las razones para obedecerlo son acordes con juicios intuitivos acerca de la rectitud del sistema de justicia penal- y no se trata de la administración del derecho que consiste en hacer que la gente obedezca normas penales a través de medios diferentes a las reglas en sí mismas, es decir, en cuanto razones (morales o prudenciales) para obedecerlas.

${ }^{48}$ Hart, H., Punishment and Responsibility. Essays in the Philosophy of Law, Oxford, Oxford University Press, 2008, p. 39, 44. 
(2) Basado en las explicaciones y predicciones de la teoría psicológica cognitiva, el AECD ofrece una serie de prescripciones de política pública encaminadas a corregir o utilizar los sesgos cognitivos de los seres humanos. El AECD utiliza una teoría cognitiva psicológica y descriptiva para llevar a cabo los objetivos normativos dentro de un marco económico. Entonces, el AECD no puede ser considerado una teoría, sino un conjunto de prescripciones sobre cómo hacer efectiva la obediencia (jurídica) del derecho y, en consecuencia, para cumplir distintas metas normativas del sistema jurídico: a saber, las razones que justifican por qué un tipo de acto es objeto de prohibiciones (por ejemplo, criminalización), obligaciones y permisiones jurídicas. De esta manera, es difícil hablar del AECD como una teoría. No se trata de una serie de axiomas para evaluar correctamente los valores de los resultados y las probabilidades de las circunstancias del mundo, ni tampoco es un sistema de valores morales de los cuales sea posible saber cómo actuar en una situación y un contexto de elección particulares. Entonces, el AECD es diferente en su naturaleza del carácter teórico de las teorías tradicionales del derecho penal invocadas por HT en su argumento.

Por otra parte, la función del derecho penal en la teoría de Duff es llamar (incluso a través de la coerción) al sospechoso de un delito a rendir cuentas ante los jueces penales. Esta función tiene que ver con la respuesta del derecho a la detección de la comisión de un delito - "a fait accompli”-, y con el problema de si se justifica, o no se justifica, la atribución de responsabilidad y, además, la imposición de una pena al presunto delincuente. La política criminal del nudge defendida por el AECD, de su parte, tiene que ver con una función diferente del derecho penal y de la pena, a saber, la de prevenir la ocurrencia del comportamiento delictivo de la gente.

La función principal del derecho penal no puede ser la de llamar a los presuntos delincuentes a rendir cuentas en el proceso penal y castigarlos si no tienen una respuesta satisfactoria para considerar que están exculpados por la acción que se ha probado que son responsables. Las funciones de imponer una pena justa y merecida, y la oportunidad de entablar un discurso racional con el delincuente en el proceso, presuponen que la función de prevenir delitos ha fallado en una primera instancia. Las primeras son 
funciones que se espera que el derecho penal cumpla -esto es incuestionable- pero solo si el derecho penal no logra cumplir la última función: "Las principales funciones del derecho como medio de control social no han de ser vistas en los litigios privados o en las causas penales, que representan provisiones vitales, pero no obstante ello accesorias, para las fallas del sistema. Han de ser vistas en las diversas formas en que el derecho es usado para controlar, guiar y planear la vida fuera de los tribunales"49.

La función del proceso penal de entablar una comunicación racional entre el defendido y el estado, no tiene la capacidad de prevenir que la gente cometa delitos. Asi como la función de prevenir delitos es incapaz de resolver la cuestión de si está justificado castigar al defendido que ofrece razones en el proceso para excusarse por lo que hizo. Además, las razones instrumentales o prudenciales que pudo tener quien ha cometido el injusto, para creer y valorar con la utilidad más alta la acción que eligió realizar, no lo justifican o lo exculpan de ser responsable de cometer un injusto: por ejemplo, "yo lo hice porque creí que la probabilidad de ser detectado era baja y la satisfacción de hacerlo era más alta que el valor que yo asigné al hecho de ser castigado".

Si se acepta el argumento retributivo de que la principal función del derecho penal y de la pena no es prevenir la ocurrencia de delitos, también es cierto que prevenir delitos es una consecuencia no-intencional de la creación de normas penales. Cuando un legislador crea una norma penal que criminaliza un tipo de acción, es irrazonable negar que el ejercicio de este poder normativo de criminalización se encamina a alterar el comportamiento de las personas para que ellas no cometan delitos, a saber, la pena tiene una función prospectiva de evitar el comportamiento criminal de la gente. Es difícil imaginar que la criminalización de un legislador de un tipo de acción no tiene, normalmente, el objetivo de evitar que la gente produzca daños a otras personas al realizar actos típicos del tipo de acción criminalizada. Es un hecho que no puede ser negado por las teorías de la pena del justo-merecimiento. Imponer una pena no solo está justificado para infligir un sufrimiento merecido al que ha cometido un injusto. La imposición del sufrimiento está justificada para evitar el sufrimiento 
La política criminal del nudge.

futuro, y no en el sufrimiento en sí mismo, sea o no con base en razones instrumentales ${ }^{50}$.

Asimismo, si se acepta la afirmación retributiva de que prevenir delitos no debe ser una razón para los jueces de imponer un castigo -a saber, imponer sufrimiento punitivo en un delincuente para prevenir a otros de cometer delitos- prevenir es un efecto inevitable de imponer una pena como parte de un proceso penal, aunque sea un resultado no-intencional. Lo mismo podría decirse de la inocuización producida con la pena (por ejemplo, con la prisión): no es el resultado intencional que quería obtener el que castiga, pero un efecto de la inocuización, así sea no intencional, consiste en evitar que el que ha sido castigado cometa futuros delitos, y es posible que también funcione para evitar que otros se mal comporten del mismo modo.

(3) También existen teorías de derecho penal tradicionales que afirman que la función del derecho penal es evitar que la gente cometa delitos y que también asumen una racionalidad de medio-a-fin de la gente, por ejemplo, la teoría de Hart. Las medidas punitivas imponen un costo que se justifica solo por el beneficio compensatorio de reducir conductas criminales futuras. El éxito del sistema de derecho penal es medido por el número de delitos no cometidos. Y la imposición de una pena se justifica porque es útil para desincentivar a la gente a desobedecer el derecho ${ }^{51}$.

La razón que la regla en sí misma ofrece para ser obedecida normalmente coincide con las razones morales o prudenciales subyacentes que tiene la gente para obedecerla: por ejemplo, porque aceptan lo que las normas jurídicas requieren o para evitar el riesgo de ser castigado o para seguir lo que los otros hacen, etc. ${ }^{52}$.

La obediencia al derecho es una práctica de seguir reglas y no es un patrón de comportamiento meramente habitual o accidental ${ }^{53}$. Entonces, se requiere una obediencia general del derecho en cuanto este es aceptado por la gente, a saber, porque lo que una norma penal requiere hacer o abste-

\footnotetext{
${ }^{50}$ Hart, H., Punishment and Responsibility, op. cit., p. 160.

${ }^{51}$ Ibíd., p. 47.

${ }^{52}$ Gardner, J., Op. cit., p. xliii.

${ }^{53}$ Hart, H., The Concept of Law, Op. cit.
} 
nerse de hacer es valioso en sí mismo, sin importar la sanción consecuente conectada por la misma norma a su desobediencia: i.e. la eficacia jurídica ${ }^{54}$. En este escenario, las amenazas son irrelevantes en el razonamiento práctico de la gente: no hay coacción para hacer que una persona haga lo que ella ya de por sí quiere hacer ${ }^{55}$. La amenaza es un motivo de obediencia de las normas penales, es decir, solo constriñe a los que, en ausencia de dicha motivación, estarían motivados a hacer lo que la norma requiere que se abstengan de hacer, o a no hacer lo que la norma requiere que hagan.

La amenaza de un castigo es un incentivo negativo para desmotivar el comportamiento criminal: si usted escoge desobedecer, usted enfrenta el riesgo de ser castigado, es decir, usted debe "pagar un costo". Pero el castigo es más que un simple incentivo negativo. Las normas penales tienen un significado normativo, a saber, que el comportamiento que es criminalizado por medio de ellas es moralmente incorrecto ${ }^{56}$; las normas están "to be taking seriously as a standard of behavior [...] Punishment is different from a mere tax on a course of conduct [...] the first involves, as the second does not, an offence or breach of duty in the form of a violation of a rule set up to guide the conduct of ordinary citizens" ${ }^{\prime \prime}$.

Que la gente desobedezca normas penales no implica que las reglas sean irrelevantes en el razonamiento práctico de las personas, sino que ellas tienen razones internas para actuar racionalmente que pesan más que las razones externas (internalizadas personalmente) para actuar conforme con lo que la regla prescribe. En otras palabras, es posible considerar que la gente obedece una norma penal porque asignan un valor moral al resultado que se obtiene por hacer, o abstenerse de hacer, lo que la norma requiere de ellos, a saber, un valor que les preocupa personalmente maximizar más que la maximización del valor de obedecer: por ejemplo, hacer lo que el derecho requiere por el hecho de ser requerido por el derecho maximiza el valor de guíar la acción por el derecho; no cometer hurto maximiza el

${ }^{54}$ Gardner, J., Op. cit., p. xliii.

55 Searle, J., Op. cit., p. 160.

56 Sunstein, C., The Ethics of Influence, Op. cit., p. 19.

${ }^{57}$ Hart, H., Punishment and Responsibility, Op. cit., p. 44; Hart, H., The Concept of Law, Op. cit., p. 39. 
valor moral de respetar la propiedad de otros. Sin embargo, es posible considerar que las razones por las que se maximiza este valor son pro tanto, a saber, razones que son derrotables: cometer hurto para maximizar un valor diferente por el cual la persona se preocupe más, por ejemplo, cometer un hurto para comprar un medicamento costoso para salvar la vida de un pariente amado. De modo que, las reglas penales son "[...] reasons for exercising choice in the direction of obedience, leaving to individuals to choose" ${ }^{\text {}}$. El delincuente puede sopesar el costo que asigna a la obediencia del derecho en contra de la satisfacción que podría obtener por desobedecerlo, ponderado por la probabilidad de no ser detectado y castigado: "if the sanctions of the criminal law are applied, the pains of punishment will for each individual represent the price of some satisfaction obtained from the breach of the law" 59 .

En este orden de ideas, es razonable afirmar que el valor de la dignidad humana implica el reconocimiento de que cada persona es un "choosing being" 60 , es decir, un individuo que cuida de sus preocupaciones personales con sus decisiones, y que está provisto de la capacidad de razonar instrumentalmente para alcanzar el propósito, representado en su intención, con la acción que elija realizar. Los individuos necesitan saber lo que los tribunales harán en el futuro, es decir, quieren saber ex ante las consecuencias materiales de sus acciones. La gente tiene la capacidad de predecir "the incidence of the public force through the instrumentality of the courts" $\mathrm{y}$, basados en esta predicción, escoger prudentemente uno de los cursos de acción alternativos ${ }^{61}$. Esta descripción instrumental de la racionalidad de la gente al obedecer el derecho, reconoce Hart, "[...] can sound like very cold, if not immoral attitude toward the criminal law, general obedience to which we regard as an essential part of a decent social order. But this attitude seems repellent only if we assume that all criminal laws are ones whose operation we approve. To be realistic we must also think of bad and repressive criminal laws; in South Africa, Nazi Germany, Soviet Russia, and no doubt

\footnotetext{
58 Hart, H., Punishment and Responsibility, Op. cit., p. 44.

59 Ibíd., p. 48.

60 Ibíd., p. 49.

61 Holmes, O. W., “The Path of the Law”, Harvard Law Review, 8, 10, 1897, pp. 459- 460.
} 
elsewhere, we might be thankful to have their badness mitigated by the fact that they fall only on those who have obtained a satisfaction from knowingly doing what they forbid"62.

Por otra parte, la función comunicativa del proceso penal y la función preventiva del nudging penal no se excluyen mutuamente. Las funciones del derecho penal de prevención, imposición de penas justificadas, y la provisión de la oportunidad de comunicación racional en el juicio penal son complementarias y congruentes al maximizar la libre elección y la libertad individual al interior de un sistema penal coercitivo. La imposición justificada de una pena presupone una relación de culpa entre un injusto y quién lo comete, por esto, existe una distinción entre personas inocentes y culpables: castigar a alguien inocente es inmoral ${ }^{63}$. Aunque el castigo injustificado es un resultado riesgoso no deseado, y pese a que su ocurrencia sea un resultado inevitable -a saber, debe ser aceptado con cierto grado tolerable de probabilidad-, el proceso es una oportunidad para verificar que no haya condiciones exculpantes, e imponer así una pena justificada al delincuente: "we look on excusing conditions as something that protects the individual against the claims of rest of society" ${ }^{\text {" }}$.

Así, las funciones de imponer un castigo justificado y la comunicación racional en el proceso son congruentes con asumir que la gente está provista de racionalidad instrumental. Estas funciones acrecientan la capacidad de las personas de hacer predicciones más confiables sobre las consecuencias de sus propias elecciones, a saber, comportarse intencionalmente de una forma y no de otra manera posible. Por ejemplo, al defendido no se le puede imponer una pena si existen defensas - "excusing conditions"- para considerar que, desde su punto de vista interno, su acción fue involuntaria: es decir, que en razón de su condición mental ella debe ser exculpada, porque sería injusto castigarla por algo que hizo sin intención ${ }^{65}$.

Tanto la función de prevención como la (no) imposición justificada de una pena como resultado de un proceso son complementarios para maximi-

62 Hart, H., Punishment and Responsibility, Op. cit., p. 47.

${ }^{63}$ Gardner, J., Op. cit., p. xviii.

${ }^{64}$ Hart, H., Punishment and Responsibility, Op. cit., p. 44. 
zar la libre elección y la libertad individual al interior de un marco coercitivo de un sistema penal ${ }^{66}$. Asumir que la gente (des)obedece lo que las reglas penales requieren, basados en el razonamiento instrumental, es congruente con la idea del estado de derecho liberal de guiar la acción humana a través de normas jurídicas, es decir, razones. Las reglas penales proveen una guía jurídica al ofrecer advertencias por adelantado sobre la imposición de un sufrimiento punitivo a aquellos que eligen cometer un delito. Así, las normas penales anuncian ciertos estándares de comportamiento y también las sanciones que están conectadas a su desviación, dejando que los individuos escojan entre obedecer o pagar ${ }^{67}$. Los individuos tienen la capacidad de planear sus propias vidas si saben cómo comportarse para no tener la interferencia de un castigo penal en sus vidas: a saber, la capacidad de evitar transgredir el derecho y, en consecuencia, la imposición de un castigo penal ${ }^{68}$.

Esto no significa que el derecho altera el comportamiento de la gente solo a través de estímulos. Más bien, el derecho penal es un "choosing system" que guía a las personas para que se comporten de un modo preciso y no de otra manera posible: "We must cease to regard the law simply as a system of stimuli goading the individual" 69 . El derecho es eficaz de una forma muy especial que es irreducible a una provocación o estimulación efectiva, a saber, el derecho es "eficaz jurídicamente": las reglas jurídicas guían a la gente: el derecho ofrece el servicio de dar reglas como razones autoritativas para actuar, es decir, si son seguidas, y no simplemente observadas, por la gente. Esto no significa que se rechace el aspecto de la efectividad del derecho entendida como la estimulación efectiva en la actividad mental de las personas, sino que el derecho debe ser obedecido generalmente en términos de eficacia jurídica, y de modo excepcional simplemente observado por la gente.

El estado de derecho, entendido instrumentalmente, es un medio para obtener distintos valores que no pueden ser maximizados en conjunto: la elección libre de las personas y la eficacia jurídica del derecho ${ }^{70}$. Por un lado, cumplir con el objetivo de acrecentar la libertad de elegir de la

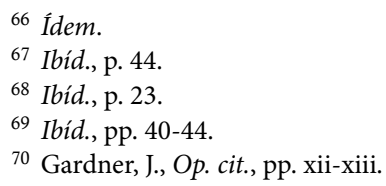


gente entre obedecer y desobedecer el derecho puede disminuir la eficacia jurídica del derecho: las reglas pueden volverse incrementalmente desobedecidas, o meramente observadas por razones prudenciales, y no seguidas como razones no-prudenciales o morales para actuar. Por otro lado, cumplir con el objetivo de acrecentar la eficacia jurídica del derecho puede llevar a que se considere el valor de la elección libre de las personas como irrelevante: si la gente obedece el derecho siguiendo una norma como una guía, o como una razón no-prudencial para actuar, desobedecer el derecho dejaría de ser una opción. Y acrecentar la eficacia del derecho a través de un sistema de estímulos que haga a la gente reaccionar conforme a lo que las reglas penales exigen podría producir el peor resultado posible, esto es, disminuir tanto la capacidad de elegir libremente de la gente, como la eficacia jurídica del derecho: a saber, convertir la libertad de elección y el seguimiento de una norma en algo que no es una opción. Por una parte, dirigir a la gente a creer que escogieron obedecer una norma penal, ya sea por razones prudenciales (es decir, que están escogiendo observar el derecho), o bien, porque la están aceptando como una guía, es decir, como una razón autoritativa para actuar. Por otra parte, dirigir a la gente a no ser consciente de que están siendo condicionados causalmente para comportarse de cierta manera particular y no de otra forma opcional, es decir, siendo inconscientes de que están, de hecho, reaccionando a los estímulos producidos en sus mentes por una elección consciente del regulador de la "arquitectura", sin importar el contenido de significado de las reglas, es decir, independientemente de alguna razón (prudencial o de otro tipo) para actuar del modo en el que la regla lo requiere.

Sin embargo, la eficacia de la obediencia del derecho no es el único propósito del derecho penal. Hay más en juego que incrementar el grado de eficacia (jurídica) del derecho. Entonces, se debe aceptar que un objetivo debe ser sacrificado para cumplir con otro objetivo jurídico. El reconocimiento de condiciones exculpantes se justifica incluso si la eficacia de las amenazas disminuye $\mathrm{e}^{71}$. 
Para concluir, el problema normativo planteado por HT se relaciona con un desacuerdo teórico tradicional acerca de las razones que justifican la imposición de un castigo penal, a saber, por razones de merecimiento o bien por razones de prevención.

Así, el futuro del AECD no es el único que depende de cómo lidiar con este problema, sino que el futuro de cada una de las teorías del derecho penal estaría desafiada por esta cuestión normativa. Sin embargo, me parece que es injustificado este problema de incongruencia entre al AECD y las teorías retribucionistas, como también sería injustificado afirmar que el futuro de las teorías tradicionales de retribución (y la teoría de la comunicación racional de Duff) depende de cómo se lidie con la incongruencia normativa que se tiene con las teorías preventivas. Más bien, el futuro del AECD en el derecho penal depende de cómo encaje con las teorías tradicionales de prevención que no consideran que cualquier medio para lograr la mayor prevención es justificado, es decir, que prevenir delitos tiene limitaciones en términos preventivos: i.e. maximizar la libertad de la gente al interior de un sistema coercitivo. En consecuencia, es posible justificar la prevención en términos no-consecuencialistas.

\section{Conclusiones}

Las ciencias conductistas ponen en evidencia el hecho de que cada norma penal presupone una elección de la arquitectura del problema de decisión. Así pues, esta evidencia conductista deja al creador de políticas públicas en una posición difícil; saber o ser consciente de que la elección de la arquitectura es una herramienta gubernamental que afecta el comportamiento de las personas, crea una carga de justificación para los creadores de políticas; ellos deben justificar por qué enmarcan el problema de decisión de una forma y no de otra posible; deben prestar atención a lo que ellos eligen y a cómo enmarcar el problema de decisión, adicionalmente, deben justificar, en términos de ventajas sociales y costos, por qué se prefiere una enmarcación de la información a otra alternativa. No hay manera de escapar de 
ello. De esta manera, la pregunta no es si se justifica el nudging penal, sino cómo aplicar el nudging penal de una forma permisible y útil.

La aplicación de los instrumentos de política del AECD se justifica si, y solo si, se utiliza para "bien", a saber, acciones de control social de los gobiernos que produzcan la maximización, o que no la disminuyan, de la libertad y de la protección de la libre elección de las personas en un sistema coercitivo. De este modo, el reto está en concebir una teoría del derecho penal que esté basada en las explicaciones y predicciones que dejan en claro que el comportamiento de las personas está moldeado por ideales normativos que ellos apoyan conscientemente (por ejemplo, el ideal político del estado de derecho liberal), combinado con tendencias psicológicas o procesos inconscientes que se llevan a cabo en la mente de las personas sin intención deliberativa alguna ${ }^{72}$. Este reto implica superar, en la medida de lo posible, algunos desafíos para las teorías del derecho penal tradicionales. De ello depende algún grado de éxito futuro de estas teorías: a saber, su capacidad de adaptarse a lo que se conoce, o podría conocerse, sobre la psicología humana, es decir, a las "[...] new beliefs about the human mind"73.

Las políticas del nudging podrían servir a la maximización de la libertad individual, al enmarcar el problema de decisión a la gente de tal forma que ellos decidan con el mayor grado de libertad posible. Asimismo, incluso si se asume que la función principal del derecho penal es llamar a los presuntos delincuentes a rendir cuentas ante los jueces penales, el nudge es útil para lograr este objetivo. La elección de la arquitectura o de enmarcación de los problemas de decisión de los fiscales, jueces y jurados, con la intención de maximizar lo que la teoría de Duff valora más -es decir, establecer una comunicación racional entre el delincuente y el estado y pasar de la responsabilidad al castigo justificado- parece ser permisible. Los nudges podrían ser útiles para alcanzar el propósito de la función de rendir cuentas en juicio por parte de los delincuentes: enmarcar el contexto de elección de los jurados, los jueces, los abogados y la gente en general, para hacerlos cumplir con las normas procesales, apelando a su proceso mental cognitivo

72 Shafir, E., "Introduction and Biography", en Tversky, A., Preference, Belief, and Similarity, Shafir, Eldar. (Ed.), Cambridge, Massachusetts, MIT Press, 2004, p. ix. 
y automático y, por lo tanto, con el propósito de establecer una comunicación racional entre el delincuente y el estado. Ahora bien, pregunto: ¿son objetables estos nudges procedimentales por el hecho de que los creadores de políticas traten a la gente como autómatas?

Adicionalmente, si el nudging es ubicuo, existen razones para creer que es razonable considerar permisible que los gobiernos utilicen esta herramienta para contrarrestar las elecciones arquitecturales hechas por personas con la intención de utilizar a otras personas como un medio para lograr el objetivo personal de un arquitecto privado, es decir, una influencia manipuladora y engañosa sobre las elecciones de la gente: por ejemplo, información falsa o imprecisa sobre la probabilidad de ser capturado en el aeropuerto de Bogotá al intentar salir con alguna cantidad de cocaína, que no sea consistente con las estadísticas de los injustos que en realidad han sido detectados.

\section{Bibliografía}

Bucciarelli, M. \& Johnson-Laird, P.N. "Naïve Deontics: A Theory of Meaning, Representation, and Reasoning", Cognitive Psychology, 50, 2, 2005, pp. 159-193.

Duff. R. A., "Responsabilidad y punibilidad en el derecho penal" en Kramer M., Grant C., Colburn, B. y Hatzistavrou, A. (eds.), El legado de H. L. A. Hart. Filosofía jurídica, política y moral, Madrid, Marcial Pons, 2012, pp. 145-168.

Edwards, J., "Theories of Criminal Law", en Edward N. Zalta (ed.), The Stanford Encyclopedia of Philosophy, Stanford, Stanford University, 2018, disponible en: <https://plato.stanford.edu/archives/fall2018/ entries/criminal-law/>.

Gardner, J., "Introduction" en Hart, H., Punishment and Responsibility. Essays in the Philosophy of Law, 2 ed. (2008) Oxford, Oxford University Press, 1968, pp. xiii-liii.

Green, L., "Introduction", en Hart, H., The Concept of Law, 3 ed. (2012), Oxford, Oxford University Press, 1961, pp. xv-lv. 
Hart, H., Punishment and Responsibility. Essays in the Philosophy of Law, 2 ed. (2008) Oxford, Oxford University Press, 1968.

Hart, H., El concepto de derecho, Buenos Aires, Abeledo Perrot, 1963.

Holmes, O. W. [1897]: “The Path of the Law”, Harvard Law Review, 8, 10, pp. 457-478.

Jeffrey, R., The Logic of Decision, Chicago, University of Chicago Press, 1985.

Jolls, Christine, Cass R. Sunstein, C. y Thaler, R., "A Behavioral Approach to Law and Economics", Stanford Law Review, 50, 1998, pp. 1471-1550.

Kahneman, D., Thinking Fast and Slow, London, Penguin Books, 2011.

Mongin, P. \& Cozic, M., "Rethinking nudge: not one but three concepts", Behavioral Public Policy, 2, 1, 2018, pp. 107-124.

Moreno Cruz, D., "Un punto de vista normativo acerca del "Nudge financiero" en Daniel Castaño (ed.), El derecho administrativo como idea y sus transformaciones contemporáneas, Bogotá D.C., Universidad Externado de Colombia, 2018, pp. 411-445

Moreno Cruz, D., “Three Realistic Strategies for Explaining and Predicting Judicial Decisions" en Mathis K. (ed.), Law and Economics in Europe. Foundations and Applications, Dordrecht, Springer, 2014, pp. 93-124.

Searle, J., Making the Social World. The Structure of Human Civilization, Oxford, OUP, 2010.

Shafir, E., "Introduction and Biography", en Tversky, A., Preference, Belief, and Similarity, Shafir, Eldar. (Ed.), Cambridge, Massachusetts, MIT Press, 2004, pp. ix-xv.

Sunstein, C., Kahneman, D. \& Schkade, D., "Assessing punitive damages (with Notes on Cognition and Valuation in Law)", The Yale Journal, 107, 1998, pp. 2071-2153.

Sunstein, C., The Ethics of Influence. Government in the Age of Behavioral Science, Cambridge, New York, Cambridge University Press, 2016.

Sunstein, C., "Misconceptions about Nudges", Journal of Behavioral Economics for Policy, 2, 1, 2018, pp. 61-67.

Thaler, R. \& Sunstein, C., Nudge: Improving Decisions about Health, Wealth and Happiness, London, Penguin Books, 2009. 
Tversky, A. \& Kahneman, D., “The framing of decisions and the psychology of choice”, Science, 211, 4481, 1981, pp. 453-458.

Von Wright, G., Norm and Action. A logical Enquiry, London, Routledge and Kegan Paul, 1963.

Waldron, J., "It's All for Your Own Good", The New York Review of Books, oct. 9, 2014.

Yeung, K., "Nudge as Fudge", The Modern Law Review, 75, 1, 2012, pp. 122-148 